\title{
Médiévales
}

Langues, Textes, Histoire

77 | automne 2019

Mathématiques. Savoirs et enseignements (OrientOccident)

\section{Stylus theologicus et iuridicus : la causa Jean Petit à Constance (1414-1418) et les débats sur le tyrannicide}

Stylus theologicus et iuridicus: Jean Petit in Constance (1414-1418) and the debates on tyrannicide

\section{Sebastián Provvidente}

\section{OpenEdition}

Journals

\section{Édition électronique}

URL : https://journals.openedition.org/medievales/10527

DOI : 10.4000/medievales. 10527

ISSN : 1777-5892

\section{Éditeur}

Presses universitaires de Vincennes

\section{Édition imprimée}

Date de publication : 5 décembre 2019

Pagination : 129-151

ISBN : 978-2-37924-061-4

ISSN : 0751-2708

Référence électronique

Sebastián Provvidente, «Stylus theologicus et iuridicus : la causa Jean Petit à Constance (1414-1418) et les débats sur le tyrannicide », Médiévales [En ligne], 77 | automne 2019, mis en ligne le 01 janvier 2022, consulté le 22 avril 2022. URL : http://journals.openedition.org/medievales/10527 ; DOI : https:// doi.org/10.4000/medievales.10527 
Sebastián Provvidente

\section{Stylus theologicus et iuridicus : la causa Jean Petit à Constance (1414-1418) et les débats sur le tyrannicide}

Le 23 novembre 1407 le duc d'Orléans, frère du roi Charles VI, est abattu dans la rue. L'instigateur de l'assassinat est son cousin germain, le duc de Bourgogne, Jean sans Peur ${ }^{1}$. Le 8 mars 1408, Jean Petit, docteur en théologie, prononce à l'hôtel Saint-Paul et en présence de nombreux nobles et docteurs de l'Université une Justification du duc de Bourgogne sans précédent, au sujet de la mort du duc d'Orléans. Le texte se présente sous forme de syllogisme : alors que la première partie démontre qu'il est licite de mettre à mort un tyran lorsque celui-ci est coupable du crime de lèsemajesté, la seconde cherche à montrer que le duc d'Orléans était un vrai tyran. Nous ne nous attarderons pas ici sur les détails de l'argumentation, mais seulement sur le principal but visé par le texte ${ }^{2}$ :

1. B. GuenÉE, Un meurtre, une société. L'assassinat du duc d'Orléans, Paris, 1992, p. 7-16 ; P. RAYMOND, «Enquête du prévôt de Paris sur l'assassinat de Louis duc d'Orléans (1407) », Bibliothèque de l'École des chartes, 26 (1865), p. 215-249; F. Autrand, Charles VI. La folie du Roi, Paris, 1986 ; R. FAMIGLIETI, Royal intrigue. Crisis at the Court of Charles VI (1392-1420), New York, 1986 ; B. GuenéE, La Folie de Charles VI, Paris, 2016.

2. Chronique d'Eguerran de Monstrelet, éd. L. DouËT-D'ArCQ, Paris, 1857-1862, t. 1, p. 206. Étonnement, il n'existe aucune édition critique de ce texte. Alfred Coville, dans son étude incontournable sur le tyrannicide, semble cependant avoir fait une grande partie du travail paléographique préalable à l'édition, puisqu'il décrit une bonne partie des manuscrits (A. Coville, Jean Petit. La question du tyrannicide au commencement $d u X V^{e}$ siècle, Paris, 1932, p. 133-168). Pour comprendre l'intérêt des procès contre la majesté royale et les compilations de manuscrits effectuées au cours de l'époque moderne, voir J. CHIFFOLEAU, «Le crime de majesté, la politique et l'extraordinaire. Note sur les collections érudites de procès de lèse-majesté du XVII ${ }^{\mathrm{e}}$ siècle et leurs exemplaires médiévaux », dans Y. BERCÉ éd., Les Procès politiques (XIV'-XVII siècles). Actes du colloque de Rome (20-22 janvier 2003), Rome, 2007, p. 577-662 et 616-617. Un fragment sur les degrés de majesté de la Justification a depuis peu été partiellement édité à partir du manuscrit de Wien, ÖNB, 2657, f. 12 -13 et 44 et 46v, dans J.-P. Boudet et J. Chiffoleau, « Magie et construction de la souveraineté sous le 
Il est licite à ung chascun subjet sans quelconques mandement ou commandement selon les loys moral, natural et divine, de occire ou faire occire ycellui traitre desloial et tirant, et non pas tant seulement licite, mes honorable, et meritore, maismement quant il est de si grand puissance que justice n'en peut bonnement estre faite para le sourverain.

Celui qui complote contre la «noble majesté du roi » commet ainsi une trahison et peut être accusé de crimen laesae maiestatis. Dans les commentaires de la Lex Julia de majestate, la distinction était faite entre les crimes commis contre la personne du roi et ceux perpétrés contre la chose publique $^{3}$. Ce qu'il y a de singulier dans l'argumentation de Jean Petit, c'est qu'il essaie d'assimiler le coupable du crime de laesae maiestatis à un tyran, puisque, selon sa Justification, un tyran cherche à s'approprier la puissance du royaume par son ambition personnelle. Bernhard Bess et Friedrich Schoenstedt ont déjà souligné que la singularité de l'argumentation était probablement en rapport avec l'évolution de l'idée de royauté, particulièrement marquée par sa sacralisation dans le cas français. Sans citer Bess et sans l'avoir lu, Simon Hirsch Cuttler en est arrivé à une conclusion similaire dans son analyse du cas français ${ }^{4}$.

La défense du tyrannicide élaborée par Jean Petit provoqua une grande résistance, non seulement de la part des membres de la famille du duc d'Orléans, mais aussi chez un des principaux théologiens de l'université de

règne de Charles VI », dans M. Ostorero, J.-P. Boudet, A. PARAvicini-Bagliani, Astrologie, divination et magie dans les cours (XII ${ }^{e}$-XVII $I^{e}$ siècles), Florence, 2017, p. 157-240 (j'adresse tous mes remerciements aux auteurs qui m'ont permis l'accès à leur texte avant sa parution). Karol Skrzypczak travaille en ce moment sur une édition critique de la Justification, ce qui représente à l'heure actuelle un vrai desideratum pour l'historiographie. Sur les manuscrits de la Justification, voir D. Willard, « The manuscripts of Jean Petit's Justification : Some Burgundian Propaganda Methods of the Early Fifteenth Century », Studi Francesi, 38 (1969), p. 271-280.

3. Y. Thомаs, «L'institution de la majesté », Revue de synthèse, 112 (1991), p. 331386. Sur le concept de tyrannie au Moyen Âge, voir J. MiETHKE, « Der Tyrannenmord im späten Mittelalter : Theorien über das Widerstandsrecht gegen ungerechte Herrschaft in der Scholastik », dans G. BeESTERMÖLleR et H.-G. Justenhoven, Friedensethik im Spätmittelalter. Theologie im Ringen um die gottgegebene Ordnung, Stuttgart, 1999, p. 24-48. Sur le lien entre le crimen maiestatis et la tyrannie, voir J.-P. Boudet et J.CHIFFoleaU, « Magie et construction de la souveraineté... », p. 30-48; J. ChIFFoleau, « Sur le crime de majesté médiévale », dans Genèse de l'État moderne en Méditerranée, Rome, 1993, p. 183-313 ; J. KRYNEn, L'Empire du Roi. Idées et croyances politiques en France XIII'-XV siècle, Paris, 1993, p. 345-37.

4. B. BEss, «Die Lehre vom Tyrannenmord auf dem Konstanzer Konzil », Zeitschrift für Kirchengeschichte, 36 (1916), p. 1-61 ; F. SCHOENSTEDT, Der Tyrannenmord im Spätmittalter. Studien zur Geschichte des Tyrannenbegriffs und der Tyrannenmordtheorie insbesondere in Frankreich, Berlin, 1938 ; sur l'historiographie allemande à ce sujet, voir A. FrenkEN, «Die Erforschung des Konstanzer Konzils (1414-1418) in den letzten 100 Jahren », Annuarium historiae conciliorum, 25 (1993), p. 196 ; ID., Das Konstanzer Konzil, Stuttgart, 2015, p. 225227 ; S. H. CuttLer, The Law of Treason and Treason Trials in Later Medieval France, Cambridge, 1981, p. 6-9. 
Paris, son chancelier Jean Gerson. Celui-ci semble avoir été l'un des seuls à mettre en garde contre la fragilité d'une paix qui ne serait pas fondée sur les ciments de la justice, et à avertir rapidement des risques représentés par les arguments de Jean Petit s'ils étaient transposés dans la politia ecclesiastica.

Immédiatement après les violences perpétrées au cours de la révolte de Caboche de 1413, Jean Gerson se lança dans une entreprise à laquelle il consacrerait, de façon presque obsessionnelle, les quatre années suivantes de sa vie : faire condamner en tant qu'hérétiques les thèses soutenues par Jean Petit dans sa Justification ${ }^{5}$. Dans ce contexte, Gerson considère que ce qui empêche la paix n'est pas tant l'assassinat du duc d'Orléans - qui d'ailleurs peut être pardonné -, que l'absence de repentir chez le coupable du crime et la justification élaborée par Jean Petit, dont les thèses sont considérées comme fausses, subversives et scandaleuses. En cherchant à défendre dans sa Justification les exceptions aux principes de Non occides et non perjurabis, Jean Petit pousse les sujets à la désobéissance, à la rébellion et à la sédition ${ }^{6}$. Ses thèses font scandale, dans la mesure où elles engendrent, chez d'autres, les conditions propices au péché. Puisqu'elles ont dépassé le milieu universitaire et le cadre des disputationes, elles sont devenues un scandalum ${ }^{7}$.

Les efforts de Gerson pour faire condamner les thèses de Jean Petit provoquèrent une grande résistance au sein du milieu universitaire. Finalement, le 7 octobre 1413, le roi ordonnait à l'évêque de Paris et à l'inquisiteur, sous la direction des plus grands maîtres de l'Université, d'enquêter sur les erreurs et les hérésies. C'est ainsi que le 30 novembre 1413, se réunissait le «Concile de la foi » dont les sessions dureraient jusqu'au 23 février $1414^{8}$. En dépit des difficultés rencontrées, Gerson obtient du Concile, le 23 février 1414, la condamnation, au nom de l'évêque et de l'inquisiteur, de ces thèses. Puis, le 16 mars, par le biais d'une lettre royale,

5. A. Coville, L'Ordonnance cabochienne de 1413, Paris, 1891.

6. B. GuENÉE, «Non perjurabis. Serment et parjure en France sous Charles VI », Journal des savants (1989), p. 241-257.

7. B. GuEnÉe, Un meurtre, une société..., p. 236, et, cité par l'auteur, JEAn Gerson, Opera omnia, éd. L. E. Du Pin, Amberes, 1706, t. V, p. 244-252, 278-280 ; Jean Gerson, Euvres complètes, éd. P. Glorieux, Tournai, 1960-1963, t. X, p. 232. Sur la notion de scandalum, voir A. Fossier, «Propter vitandum scandalum. Histoire d'une catégorie juridique (XII ${ }^{\mathrm{e}}-\mathrm{XV}^{\mathrm{e}}$ siècles) », Mélanges de l'École française de Rome. Moyen Âge, 121/2 (2009), p. 317-348 ; et sur les notoria, voir J. CHIFFOLEAU, « "Ecclesia de occultis non iudicat”. L'Église, le secret, l'occulte (du XII ${ }^{\mathrm{e}}$ au XV $\mathrm{V}^{\mathrm{e}}$ siècle) », Micrologus, 14 (2006), « Il segreto nel Medioevo », p. 359-481.

8. Chartularium Universitatis Parisiensis, éd. H. Denifle et E. Chatelain, Paris, 1897, t. IV. Pour une analyse du «Concile de la foi », voir B. GuEnÉE, Un meurtre, une société..., p. 239-256. Sur le type d'assemblées au cours de la période conciliaire, voir H. MiLLET, « Du conseil au Concile (1395-1408). Recherche sur la nature des assemblées du clergé en France pendant le Grand Schisme d'Occident », Journal des savants, (1985), p. 137-159. Sur la violence et le contexte universitaire, voir B. SÈre, Les Débats d'opinion à l'heure du Grand Schisme. Ecclésiologie et politique, Turnhout, 2016, p. 351-592. 
la sentence était annoncée à tous les évêques. Il leur était alors ordonné de brûler toutes les copies de la Justification ${ }^{9}$. Sitôt la sentence connue, le duc de Bourgogne appelait le futur concile général à se réunir à Constance à partir du $1^{\text {er }}$ novembre 1414 afin de résoudre le Schisme. Cet appel était renouvelé en septembre $1414^{10}$.

Notre étude se propose ici d'analyser les discussions entre théologiens et juristes à propos de la Justification du tyrannicide de Jean Petit dans le cadre du Concile de Constance. Mais il nous paraît nécessaire de proposer, au préalable, un nouveau récit de ce procès fondé sur l'étude de certains textes qui n'ont guère reçu de la part de l'historiographie l'attention qu'ils méritent $^{11}$.

\section{Quilibet tyrannus : une victoire à la Pyrrhus}

Le procès hors de la sphère française commence avec l'appel du duc de Bourgogne en mars 1414. Jean XXIII, pape de l'obédience pisane, délègue alors l'affaire aux cardinaux Orsini, Panciera et Zabarella, et ce sont eux qui, par la suite, citent l'évêque et l'inquisiteur de Paris ${ }^{12}$.

Alors que la paix d'Arras a permis que l'affaire ne devienne pas une priorité pour la nation gallicane à Constance, et tandis que les deux camps se sont engagés à ne pas l'ébruiter devant le concile, Gerson, arrivé le 21 janvier, fait immédiatement tout son possible pour faire condamner les thèses de Petit ${ }^{13}$. Il prononce peu après le fameux sermon Ambulate dum lucem habetis, dans lequel il expose la plupart des principes qui confèrent l'autorité au Concile. Ces derniers seront ensuite consignés dans le décret Haec sancta synodus, par le biais duquel les Pères conciliaires cherchent une échappatoire au trouble provoqué par la fuite de Jean XXIII.

9. A. Coville, Jean Petit. La question du tyrannicide..., p. 439-503.

10. A. Coville, Jean Petit. La question du tyrannicide..., p. 504-505 ; Acta Concilii Constanciensis, éd. H. Finke, Münster, 1896-1928 (4 vol.) (dorénavant ACC), t. IV, p. 240.

11. ACC : les principales sources se trouvent dans le IVe volume, p. 237-432. Voir aussi Jean Gerson, Euvres complètes, t. X (spécialement ce volume), et ID., Opera omnia, t. V. Les mss Paris, BnF, lat. 1485 I et II ont servi à Finke à éditer de nombreux textes, ce qui ne l'empêchait pas d'avertir qu'il restait de nombreux textes à publier. Ce manuscrit a apparemment appartenu à Martin Porrée, qui joua un rôle fondamental dans l'affaire Petit et fut un opposant acharné de Jean Gerson à Constance. Martin Cable a récemment attiré l'attention sur un consilium inédit du juriste italien Raffaele Fulgosio à propos de l'affaire Petit, qui présente un grand intérêt : M. J. CABLE, "Cum essem in Constantie... » Raffaele Fulgosio and the Council of Constance, Leyde, 2015, p. 268-278. Les deux autres manuscrits sur l'affaire sont Paris, BnF, lat. 1486, et Dijon, AD Côte d'Or, 11614, liasse 1, Cote 11.

12. Jean Gerson, Opera omnia, t. V, p. 501.

13. Sur la paix d'Arras, voir L. Mirot, «Autour de la paix d'Arras », Bibliothèque de l'École des chartes, 75 (1914), p. 253-327. Au-delà des termes de la paix d'Arras, Gerson insistera pour effectuer un lien entre sa position dans l'affaire Petit et les instructions reçues par le roi et l'Université : Jean Gerson, Euvres complètes, t. X, p. 208. 
Indépendamment de ces principes, Gerson semble faire référence au duc de Bourgogne lorsqu'il affirme que le Concile doit poursuivre sans exception ceux qui soutiennent des erreurs au sujet de la foi. Il déclare dans ce même texte que, pour ceux qui défendent des hérésies, une simple déclaration conditionnelle de foi ou une confession sont insuffisantes. Ils doivent réaliser une purgatio en présence d'un juge ${ }^{14}$.

D'après un mémorial bourguignon, Gerson lut le 11 avril une cedula dans la chambre de Pierre d'Ailly, où étaient réunis plusieurs membres de la natio gallicana. Dans ce mémorial, il est décrit comme enragé, jetant la cédule aux pieds du cardinal ${ }^{15}$. Ce texte sera par la suite présenté au procureur du duc de Bourgogne, afin de faire valoir que ce n'étaient pas eux qui avaient donné matière à faire condamner Jean Petit et, par conséquent, qu'ils n'avaient pas modifié les termes de la paix d'Arras ${ }^{16}$. C'est dans ce contexte, le 3 juin, que Gerson accuse précisément de crimen laesae maiestatis tous ceux qui soutiendraient la justification de l'assassinat du duc d'Orléans par le duc de Bourgogne. L'expression judiciaire de crimen laesae maiestatis n'est pas choisie au hasard, puisqu'il s'agit d'un argument important dans la Justification de Jean Petit ${ }^{17}$.

Peu après, un texte anonyme, écrit entre le 15 juin et le 5 juillet et reprenant les dires de Gerson, réclame de procéder ex officio. Auparavant, le 7 juin, Gerson s'était exprimé en présence de Pierre d'Ailly et de Francesco Zabarella sur le fait qu'il était du devoir du Concile de condamner les neuf assertions déjà déclarées hérétiques par l'inquisiteur et l'évêque de Paris. Le 8 juin, dans la résidence de Zabarella et en présence du rex Romanorum Sigismond, la question de la condamnation est soulevée par Pierre d'Ailly lui-même. Le 9, devant un grand nombre de docteurs en théologie et de canonistes, Gerson exhibe un texte qui contient les neuf assertions ${ }^{18}$. Le 14 ou le 15 juin, l'affaire est finalement confiée aux commissarii in causis fidei, commission composée des cardinaux Orsini, d'Aquilée, Zabarella et Pierre d'Ailly ainsi que de quatre autres membres, appartenant chacun à une des

14. B. P. McGuire, Jean Gerson and the Last Medieval Reformation, Philadelphie, 2005, p. 245-246 ; JEAN GERSON, Euvres complètes, t. V, p. 39-50.

15. Jean Gerson, Euvres complètes, t. X, p. 529. Voir S. Valléry-Radot, Les Français au concile de Constance. Entre résolution du Schisme et construction d'une identité nationale, Turnhout, 2016 (j'adresse tous mes remerciements à Nicole Bériou qui m'a permis de travailler sur cette thèse avant sa publication). Bien que le cas de Jean Petit n'y soit pas exclusivement abordé, cet ouvrage contient de nombreux passages intéressants sur le sujet. Sur l'affaire en particulier, voir S. VALLÉRY-RADOT, « Die Causa Jean Petit und das Problem des Tyrannenmords », dans K. Evers, Das Konstanzer Konzil. Katalog 1414-1418, Darmstadt, 2014, p. 111-115.

16. Jean Gerson, Euvres complètes, t. X, p. 529.

17. Jean Gerson, Euvres complètes, t. X, p. 534.

18. Jean Gerson, Euvres complètes, t. X, p. 530-531. Le 12 juin, devant quelques cardinaux et ambassadeurs et devant Sigismond en personne, il soulevait encore une fois la question. 
nations du Concile ${ }^{19}$. Martin Porrée, évêque d'Arras et défenseur du duc de Bourgogne, contestera rapidement l'intégration d'un ennemi déclaré tel que Pierre d'Ailly au sein de la commission ${ }^{20}$.

Le 24 juin, Sigismond quitta Constance pour Nice afin de négocier l'abdication de Benoît XIII. Avant son départ, il avait menacé de ne revenir à Constance qu'une fois la causa Petit terminée ${ }^{21}$. Aux dires de la délégation du duc de Bourgogne, ses ennemis croyaient pouvoir obtenir en moins d'une semaine la condamnation des thèses ${ }^{22}$. C'est dans ce contexte qu'a été rédigée la proposition Quilibet tyrannus, à partir d'une liste de sept assertions que Gerson avait, sans succès, essayé de faire condamner par l'évêque de Paris, entre le 4 et le 6 septembre $1413^{23}$. Le 27 juin, au cours de la réunion des députés des nations et de la facultas theologica, Valentin Vital, l'évêque de Toulon, se faisant l'écho de la requête de Sigismond, demande aux envoyés du duc de Bourgogne de se retirer, puisque ceux-ci étaient pars in causa. Après leur départ, conformément au désir de Sigismond, l'évêque de Toulon s'attache à faire condamner l'assertion Quilibet tyrannus, dans laquelle ni le duc de Bourgogne ni Jean Petit n'étaient expressément nommés. Le lendemain, lorsque Martin Porrée apprend la condamnation, il s'empresse de demander à l'évêque de Toulon si la proposition avait un quelconque rapport avec le texte de la Justification et si elle portait atteinte à l'honneur du duc.

Ce qui ressort des textes discutés dans cette affaire, c'est que la première des assertions de septembre 1413 est beaucoup plus radicale que celle acceptée par le Concile de la foi. Toutefois, le début du décret conciliaire retenait la radicalité de la première formulation et l'idée qu'une déclaration judiciaire n'était pas nécessaire pour procéder contre un tyran ${ }^{24}$.

Dans son homélie Prosperum iter faciet nobis Deus, prononcée après le départ de Sigismond, Gerson revenait sur les principes du décret Haec sancta qui stipulait la suprématie de l'autorité du Concile à propos de la

19. Jean Gerson, Euvres complètes, t. X, p. 531 ; W. Branduüller, Das Konzil von Konstanz (1414-1418), 2 vols, Paderborn, 1991, 1, p. 386 ; Sacrorum conciliorum nova et amplissima collectio, éd. J. D. MANSI, Florence/Venise, 1759 (Reproduction typographique 1961), p. 27, 728.

20. W. Brandmüller, Das Konzil von Konstanz..., p. 386. À propos de Martin Porrée, voir S. VALLERY-RADOT, Les Français à Constance..., p. 262-265.

21. ACC, t. II, p. 41. Au début du Concile, les relations entre Sigismond et le duc de Bourgogne étaient très tendues et le rex Romanorum tentera de l'affaiblir territorialement. Ceci explique dans une certaine mesure l'attitude hostile du duc au commencement de l'affaire Petit. Sigismond s'appuiera, au sein de la natio gallicana, sur Jean de Marroux et essaiera d'imposer son agenda, voir S. VALLERY-RADOT, Les Français à Constance..., I, p. 186.

22. JeAn Gerson, Euvres complètes, t. X, p. 531 ; ID., Opera omnia, 5, p. 383.

23. JeAn Gerson, Opera omnia, t. V, p. 56.

24. Jean Gerson, Opera omnia, t. V, p. 274 ; Conciliorum Oecumenicorum Decreta (dorénavant COD), éd. G. Alberigo et al., Bâle, 1962, p. 408. 
sortie du Schisme, de l'extirpation de l'hérésie et de la réforme de l'Église. Dans le domaine de l'hérésie, le Concile avait déjà montré son autorité lors du procès contre Jean XXIII et de la persécution de Jean Hus en ne faisant pas de favoritisme. Bien qu'ils aient compté d'importants défenseurs au sein du Concile, tous deux avaient été punis ${ }^{25}$.

Lors de la session du 6 juillet, punissant de mort Jean Hus, les Pères conciliaires condamnèrent la proposition Quilibet tyrannus... Mais cette décision qui ne touchait que l'une des sept thèses extraites par Gerson, et non les neuf condamnées à Paris en 1413, ne pouvait satisfaire le chancelier, pas plus qu'elle ne répondait aux attentes des défenseurs du duc de Bourgogne qui souhaitaient une annulation définitive. Loin de mettre fin à l'affaire Petit, cette décision suscita la rédaction de nombreuses cédules, protocoles notariaux et plusieurs consilia de juristes ${ }^{26}$.

\section{Tous à l'offensive}

Peu de temps avant la sentence contre la proposition Quilibet tyrannus, Pierre Cauchon, une des pièces maîtresses de la délégation du duc de Bourgogne, laissait entrevoir sa stratégie ${ }^{27}$. Dans un texte daté du $1^{\text {er }}$ juillet 1415, il remettait en question la façon de procéder du Concile et affirmait que ce dernier n'aurait pas dû agir ex officio, puisqu'une partie avait déjà assumé le rôle de dénonciateur. Cauchon déclarait de même qu'il n'avait pas été établi avec certitude que la question des assertions de Petit était une materia fidei ${ }^{28}$. Ce sera dorénavant un des principaux arguments développés au cours des débats de Constance. Le 2 septembre, Pierre d'Ailly répondait qu'au contraire les thèses de Petit concernaient des principes de foi ${ }^{29}$. Dans ce contexte, les partisans du duc de Bourgogne se faisaient un plaisir d'affirmer que la proposition condamnée par le Concile n'avait pas été formulée identiquement à celle de Jean Petit. Ils essayaient également d'obtenir les témoignages de la Faculté de droit canonique et de la natio picarde, puisque la condamnation de 1413 avait été prononcée en dépit de leur commune opposition et dans un climat marqué par l'absence d'unanimité sur ce sujet ${ }^{30}$. Leur stratégie consistait en même temps à obtenir

25. B. P. McGuire, Jean Gerson and the Last Medieval Reformation..., p. 245-246 ; JeAn Gerson, Euvres complètes, t. V, p. 471-480.

26. COD, p. 408.

27. À propos de Pierre Cauchon, voir S. VAlLERY-Radot, Les Français à Constance..., p. 271-273. ; F. Neveux, L'Évêque Pierre Cauchon, Paris, 1987. Sur ses actions au cours du procès contre Jeanne d'Arc, voir J. Chiffoleau, « L'hérésie de Jeanne. Note sur les qualifications dans le procès de Rouen », dans J.-P. Boudet et X. Hélary, Jeanne d'Arc. Histoire et mythes, Orléans, 2014, p. 13-55.

28. ACC, t. IV, p. 264.

29. ACC, t. IV, p. 264.

30. Jean Gerson, Opera omnia, t. V, p. 372-376. 
l'appui du roi, tout en montrant que depuis la paix d'Arras, ce dernier considérait le duc comme un bon vassal ${ }^{31}$. Au début de l'année suivante, les Gersonistes se firent pourtant un plaisir de montrer les lettres royales des 9, 10 et 16 janvier 1416, dans lesquelles le roi s'évertuait à défendre, comme si elle était sienne, l'affaire de la condamnation de Jean Petit. Il est clair que tous deux partis cherchaient à montrer qu'ils disposaient de l'appui du roi dans le contexte de l'assemblée conciliaire ${ }^{32}$.

En septembre 1415, Gerson écrit le texte Nova positio, contre ceux qui soutenaient que la condamnation de propositions philosophiques et morales n'était pas du ressort du Concile. Il y affirme également qu'il n'est pas toujours nécessaire pour condamner des erreurs de nommer une personne en particulier, surtout lorsqu'il s'agit d'individus puissants. Faisant certainement allusion au duc de Bourgogne, il affirme inconcevable que l'affaire Petit soit traitée par trois cardinaux disposant de faibles connaissances théologiques, alors que les assertions avaient déjà été analysées par de nombreux maîtres à Paris ${ }^{33}$.

Le mois d'octobre fut déterminant dans l'évolution de l'affaire, puisque le 24, la Commission de la foi citait l'évêque et l'inquisiteur de Paris à témoigner dans l'affaire de l'appel qui était toujours à la charge des trois cardinaux ${ }^{34}$. La balance paraissait maintenant incliner du côté du duc, qui n'avait pas lésiné sur les dépenses en offrant de tous côtés du vin et de l'argent ${ }^{35}$. Il n'est pas surprenant que dans un tel contexte, Gerson ait déchargé sa furie contre les ordres mendiants qu'il croyait voir œuvrer dans l'ombre ${ }^{36}$, tandis que l'ambassade du duc passait à l'offensive contre lui, en présentant, via Martin Porrée, une liste d'articles qu'il considérait hérétiques ${ }^{37}$. Celle-ci comporte plusieurs extraits de la participation de Gerson à la condamnation de Jean Hus. Tout d'abord, ce dernier était accusé d'avoir nié la proposition fides in uno solo selon laquelle, conformément à la promesse du Christ, la foi n'abandonnerait jamais son Église et pouvait d'ailleurs n'habiter qu'une seule personne, même s'il s'agissait d'une

31. Ibid., p. 388.

32. Ibid., p. 499.

33. B. P McGuire, Jean Gerson and the Last Medieval Reformation..., p. 251-252 ; JEAN Gerson, Euvres complètes, t. VI, p. 146. Sur ce sujet, il est important de remarquer que Gerson se prévaudra de la pratique de pronunciatio pour faire connaître son texte à un plus large public dans le cadre du Concile. Cette pratique consistait à établir un jour et un lieu où le texte serait lu et copié par tous ceux que cela intéresserait. Sur ce thème, consulter également D. Hobbins, Authorship and Publicity Before Pint : Jean Gerson and the Late Medieval Transformation of Learning, Philadelphie, 2009, p. 152-182.

34. A. Coville, Jean Petit. La question du tyrannicide..., p. 532.

35. ID., «Les vins de Bourgogne au Concile de Constance », Le Moyen Âge (1899), p. 326-330. Sur la pratique qui consistait à remettre de l'argent dans le cadre des procès à Constance, voir $A C C$, t. IV, p. 56.

36. Jean Gerson, Opera omnia, t. V, p. 627.

37. Jean Gerson, Euvres complètes, t. X, p. 220-225. 
femme ou d'un laïc, comme cela s'était produit avec Marie restée seule au pied de la croix ${ }^{38}$. Dans un contexte marqué par la lutte contre l'hérésie dans la causa fidei, le caractère hautement polémique contenu par cette affirmation est patent, ainsi que les conséquences anarchiques que Gerson croyait y déceler. D'un autre côté, on imputait à tort au chancelier d'avoir déclaré que, si Hus avait eu un avocat, il n'aurait pas été condamné. C'est en fait Pierre de Versailles qui aurait employé ces mots dans le cadre de l'affaire Falkenberg ${ }^{39}$. Gerson répondit à ces accusations in extenso le 30 novembre, en montrant la malveillance avec laquelle ces articles avaient été rédigés ${ }^{40}$. Puis il passa lui aussi à l'offensive. Le 21 octobre, dans le sermon Oportet haereses esse, il réfutait systématiquement les neuf assertions de Petit et mettait en garde contre les risques encourus à ne pas les condamner comme hérétiques, puisque lorsqu'il n'y avait pas de iuramentum, ceci se répercutait in subversionem tortis reipublicae ${ }^{41}$. Il était impératif que l'hérésie académique soit sanctionnée à la fois par la corporation universitaire et par le pouvoir épiscopal, et il attaquait d'un même jet l'habitude prise de soumettre toutes les affaires au Saint-Siège qui comptait peu de théologiens ${ }^{42}$. Le 29 octobre, le chancelier présentait deux textes brefs : les De protestatione circa materiam fidei et Considerationes duodecimae de pertinacia, qui expliquaient l'importance des signes extérieurs dans la détermination de l'hérésie, tout en reconnaissant la maxime ecclesia de occultis non iudicat. Ce sont précisément les signes extérieurs qui permettaient d'établir la violenta praesumptio d'un comportement hérétique ${ }^{43}$.

38. Jean Gerson, Euvres complètes, t. X, p. 220-225. Dans l'article n ${ }^{\circ}$ 7, Martin Porrée accusait Gerson de nier le principe de fides in uno solo, défendu entre autres par Ockham. La réponse de Gerson est intéressante car elle fait allusion au fait qu'il avait contesté ce principe dans le cadre de l'affaire de Hus, lorsque le maître tchèque avait soutenu que l'Église continuait d'être gouvernée sans le pape ; Jean Gerson, Euvres complètes, t. X, p. 227. À propos de Jean de Ségovie, sur le même thème, également dans un contexte conciliaire (Bâle), voir J. D. MANN, «A Conciliarist's Opposition to a Popular Marian Devotion », dans G. Christianson, T. Izbicki, C. Bellitto éd., The Church, the Councils, and Reform : The Legacy of the Fifteenth Century, Washington D. C., 2008, p. 212-226.

39. La réponse de Gerson se trouve dans la Summaria responsio ; Jean Gerson, CEuvres complètes, t. X, p. 226-230. À propos de Pierre de Versailles et de cette affirmation, voir ACC, IV, p. 352. Sur Pierre de Versailles, voir S. VAllery-Radot, Les Français à Constance..., p. 254.

40. JeAn Gerson, Euvres complètes, t. X, p. 226-230.

41. B. P. McGuire, Jean Gerson and the Last Medieval Reformation..., p. 253 ; JEAN Gerson, CEuvres complètes, t. V, p. 427.

42. Jean Gerson, Euvres complètes, t. V, p. 430-432.

43. B. P. McGuire, Jean Gerson and the Last Medieval Reformation, p. 252-253 ; JEAN Gerson, CEuvres complètes, t. V, p. 158-165 ; 5, p. 164-167. À propos du concept d'hérésie, voir les textes suivants cités dans le De protestatione circa materiam fidei : Jean Gerson, Euvres complètes, t. V, p. 164-165 ; J. CHIFFOLEAU, « "Ecclesia de occultis non iudicat"... », p. 34 . 
Novembre et décembre 1415 furent pleins de rebondissements. D'une part, la défaite des Français contre les Anglais à la bataille d'Azincourt affecta le déroulement de l'affaire à Constance, puisque Sigismond essaya de sceller une alliance avec les Bourguignons et les Anglais, modérant par là même son ardeur à faire condamner les thèses de Jean Petit ${ }^{44}$. D'autre part, le 8 novembre, les deux camps, gersoniste et bourguignon, tombèrent d'accord pour consulter l'avis des maîtres au sujet des assertions de Jean Petit ${ }^{45}$.

\section{La causa Petit : entre théologiens et juristes}

Dans ce contexte, l'affaire Petit donna naissance à des débats, au sein de la natio gallicana, non seulement sur les conséquences théologiques des assertions, mais aussi sur leurs répercussions juridiques. Une cédule rédigée peu de temps auparavant laissait déjà entrevoir les principaux arguments du camp bourguignon. D'après ses représentants, la sentence de l'évêque de Paris était nulle proprio iure, puisque ce dernier ne détenait pas le pouvoir pour juger une question de foi qui n'aurait pas été préalablement sanctionnée par l'Église. En le faisant, un évêque établissait un nouvel article de foi, ce qui était inapproprié ${ }^{46}$. Le 7 novembre, peu de temps avant que le Concile ne consulte les docteurs à propos des neuf assertions, Gaspard de Pérouse, dans un consilium, affirmait que ces neuf assertions s'appuyaient sur le droit civil et devaient être considérées comme légitimes. En associant la tyrannie au crimen laesae maiestatis - tout comme avait essayé de le faire Jean Petit - l'argumentation devenait éminemment juridique. Selon Gaspard de Pérouse, celui qui essaie d'empêcher la dominacionem principium commet un crimen lese maiestatis et doit être traité comme hostes imperii ${ }^{47}$. Comme tout bon juriste, il faisait non seulement référence au ius civile, mais aussi au ius canonicum, et citait la décrétale De clerico excommunicato qui stipulait qu'en l'absence de juges il était licite de procéder dans le but d'éviter le scandale ${ }^{48}$. Au-delà des nombreux exemples historiques romains, l'argumentation reposait juridiquement sur ces deux sources.

Au cours des mois de novembre et décembre 1415, les neuf assertions sont analysées par les docteurs. Bien qu'il ne s'agisse que d'une opinion

44. Sur les changements de politique de Sigismond, voir S. Vallery-Radot, Les Français à Constance ..., p. 289-331. Sur la guerre civile, voir B. SCHNERB, Les Armagnacs et Bourguignons. La maudite guerre, Paris, 1988.

45. Jean Gerson, Opera omnia, t. V, p. 653.

46. ACC, IV, p. 283. Sur le symbolum fidei, voir W. Brandmüller, Das Konzil von Konstanz..., t. II, p. 100. Il s'agirait d'une référence à la Somme de Thomas d'Aquin (II, 2q. 1art. 10c.).

47. Tout comme le démontrait l'extravaganti Henrici imperatoris connue sous le nom de Quoniam ; ACC, t. IV, p. 284-286.

48. $A C C$, t. IV, p. 284-286. 
et non d'une votation formelle, elle se répartit en 61 positions pro parte negativa - contre la condamnation - et 26 pro parte positiva - en sa faveur ${ }^{49}$. Ce fut un premier revers important pour les Gersonistes. À l'encontre des accusations de Martin Porrée, le 13 novembre et le 4 décembre, Gerson répondit longuement aux opinions des docteurs ${ }^{50}$. Dans ce contexte, Jourdain Morin soulignait qu'à l'instar de certaines questions de grammaire ou même de logique, pour ce qui concernait le iudicium fidei, les assertions sur le tyrannicide devaient être considérées de même ${ }^{51}$. Au début de l'année suivante, le 8 janvier 1416, Gerson demanda la réouverture de la discussion sur les assertions par un groupe moins nombreux ${ }^{52}$.

Le 16 janvier, la sentence est déclarée nulle en raison d'un vice de procédure, puisque ni l'évêque ni l'inquisiteur ne se sont présentés à Constance, bien que cités à comparaître. Dans la défense de la sentence publiée peu de temps après, les cardinaux déclaraient que la non-comparution d'une des parties leur enlevait toute possibilité de défense et que ceci allait à l'encontre du droit nature ${ }^{53}$. Outre ces arguments qui ressemblaient fort à une sortie élégante afin de ne pas avoir à se prononcer sur le fonds de la question, les trois cardinaux défendaient leur sentence en alléguant que ni l'inquisiteur de Paris ni l'évêque ne pouvaient déterminer une question de foi sans que cela ne provoque confusion et division au sein de l'Église ${ }^{54}$. D'après un texte édité par Finke et écrit en décembre 1415, si les cardinaux Zabarella et Orsini avaient proposé de repousser la décision à un futur concile, c'était parce que la sentence qui déclarait la nullité de celle de 1413 sur la base d'une erreur procédurale, n'était qu'une façon d'éviter le conflit, alors qu'une décision sur le contenu des thèses menaçait de le faire éclater ${ }^{55}$. Une fois connue, la sentence fut rapidement contestée par les Gersonistes, qui confièrent à Simon de Teramo le soin d'analyser les fautes procédurales ${ }^{56}$. L'un des arguments remettait en question le fait que les trois cardinaux auraient pris perpetuam iurisdictionem dans cette affaire, puisqu'ils avaient simplement émis des citations pour l'évêque et l'inquisiteur de Paris sans mener à bien leur

49. A. Coville, Jean Petit. La question du tyrannicide..., p. 540 ; W. BrandmüLler, Das Konzil von Konstanz..., p. 103 ; ACC, t. IV, p. 244-247.

50. JeAn Gerson, Euvres complètes, t. X, p. 230-232, 232-253.

51. ACC, t. IV, p. 297.

52. Jean Gerson, Euvres complètes, t. X, p. 254.

53. JeAn Gerson, Opera omnia, t. V, p. 586, 606-607.

54. Ibid., p. 586.

55. $A C C$, t. IV, p. 288-289.

56. Quelques-unes des principales critiques de Simon de Teramo figuraient déjà dans une série d'allegaciones et dubia sur la sentence : ACC, t. IV, p. 302-303. À propos de Simon de Teramo, voir W. BrandmüLler, «Simon de Lellis de Teramo. Ein Konsistorialavokar auf den Konzilien von Konstanz und Basel », Annuarium Historiae Conciliorum, 12 (1980), p. 229268 ; sur son activité à Constance, voir aussi W. Brandmüller, Das Konzil von Konstanz..., t. II, p. 108-109. 
exécution ${ }^{57}$. Qui plus est, la déposition de Jean XXIII avait été l'équivalent d'une mort civile et, par conséquent, le fait que les trois cardinaux n'aient pas établi leur juridiction avant sa déposition mettait en cause la légalité de leur mode de procéder ${ }^{58}$. Sur ce point, Simon de Teramo se fit un plaisir de rappeler que le cardinal Zabarella, l'un des trois cardinaux qui était à cette époque son propre maître, avait enseigné que la juridiction ne pouvait être établie simple acte de citation ${ }^{59}$. En réponse à ces critiques, Agostino del Lante omettait de mentionner l'objection procédurale selon laquelle non seulement la citation à comparaître, mais aussi son exécution effective, étaient une condition nécessaire pour exercer une juridiction dans un procès particulier. Ardicino della Porta, quant à lui, donna une réponse beaucoup plus sophistiquée ${ }^{60}$. Il importait peu que la citation ait été réellement exécutée ou non, puisque l'approbation du décret Haec sancta qui garantissait la légalité des trois juges était survenue entre temps. D'après Ardicino, la confirmation de tous les juges délégués avait été déclarée per constitutionem concilii, avant même que leur juridiction n'ait été formellement établie. Selon la démonstration de Martin Cable, il s'agirait du décret Haec sancta qui cherchait à garantir, du moins temporairement, l'exercice de la juridiction conciliaire à la suite de la déposition de Jean XXIII ${ }^{61}$. L'autre grand axe de la discussion entre Simon de Teramo et Agostino del Lante et Ardicino della Porta portait sur la manière de procéder de l'évêque de Paris et de l'inquisiteur ${ }^{62}$. D'après Simon de Teramo, certaines autorités locales - des évêques et des archevêques secondés par les universités - avaient, selon la coutume, identifié et sanctionné des hérésies non encore condamnées par l'Église dans leur ensemble. À l'instar de Jean

57. ACC, t. IV, p. 297-299. Heinrich Finke, au moment d'éditer les documents sur l'affaire Petit, n'était pas particulièrement intéressé par les conséquences techniques du procès, c'est pourquoi ces aspects n'y sont pas expliqués en détail. On ne comprend en effet pas le problème discuté par Simon de Teramo. Sur cette polémique, voir M. J. CABLE, « Haec sancta and the Continuity of Judicial Process at the Council of Constance : A Comparison of the Jean Petit Tyrannicide Case and the Dispute for me master-generalship of the Crociferi at Bologna », Annuarium Historiae Conciliorum, 40 (2008), p. 431-470. Dans ce texte, l'auteur a attiré l'attention et analysé le ms. de Graz (UB, 356) qui présentait 96 affaires légales traitées, avant et après le Concile de Constance, par quelques avocats consistoriaux. L'argument de Teramo consistait à soutenir qu'il ne suffisait pas qu'une personne soit citée à comparaitre pour établir une juridiction sur une affaire légale, mais que les citations devaient être préalablement exécutées.

58. ACC, t. IV, p. 297-299.

59. Graz, UB, ms. 356, f. 8. Texte cité par M. J. CABLE, « Haec sancta and the continuity of judicial process....», p. 438-439.

60. M. J. CABLE, « Haec sancta and the continuity of judicial process... », p. 442.

61. Graz UB, ms. 356, f. 207v. Texte cité par M. J. CABLE, « Haec sancta and the Continuity of Judicial Process... », p. 443. Sur les relations entre le Haec sancta et les causae fidei, plus particulièrement sur l'affaire de Hus, voir S. Provvidente, « La causa Hus entre plusieurs traditions académiques : conciliarisme, studia ussitica et pratiques juridiques dans le Moyen Âge tardif », Annuarium Historiae Conciliorum, 47 (2015), p. 131-152.

62. ACC, t. IV, p. 302-303. 
Gerson, Simon de Teramo affirmait que, de manière identique, les doctrines de Wyclif et de Hus avaient d'abord été sanctionnées par les autorités locales et que leur condamnation n'avait été entérinée que postérieurement par le Concile de Rome de 1413, puis par celui de Constance ${ }^{63}$. De ces exemples, découlait l'idée que, dans l'affaire Jean Petit, il fallait procéder d'une façon analogue, en confirmant les sanctions antérieures des autorités épiscopales, conformément au contenu des studia generalia. Pour sa part, Ardicino della Porta s'empressait de questionner ce droit coutumier, en invoquant le fait qu'il n'existait aucune prescription stipulant que les autorités locales possédaient la juridiction sur les questions doctrinales, celle-ci étant du seul ressort du pape, afin d'éviter ainsi la division et le schisme ${ }^{64}$.

Dans le cadre de ces débats, Raffaele Fulgosio, l'avocat consistorial du Concile de Constance, écrivit lui aussi, après son départ et de retour à Padoue, un consilium sur les neuf assertions ${ }^{65}$. Selon Cable, le terminus post quem non pour la datation du consilium est octobre 1416, puisque après cette date, l'un des signataires du texte, Benedetto Dottori, disparaît des registres de la faculté ${ }^{66}$. On ne sait pas avec certitude qui avait chargé Fulgosio de rédiger le texte, mais il est possible que ce soit quelqu'un du camp bourguignon, à l'occasion des débats ouverts à partir du 8 novembre ${ }^{67}$. Son principal apport réside dans son analyse de la première assertion de Jean Petit, qui établit qu'un sujet, sans aucun mandat, peut éliminer un tyran qui conspirerait contre le roi. Un tel passage à l'acte, non seulement serait licite, mais encore honorable et méritoire ${ }^{68}$. Martin Porrée avait effectivement fait allusion à la question du mandat en décembre 1415, lorsqu'il mentionnait que si l'empereur Sigismond procédait contre la première assertion de Jean Petit, il contredirait la législation de son prédécesseur, Henri VII. Il faisait référence au texte Qui sint rebelles, approuvé par l'empereur en 1312, au moment de son affrontement avec Robert de Naples ${ }^{69}$. Selon l'évêque d'Arras

63. Ce fut l'un des principaux arguments brandis par les Gersonistes. L'intérêt de cette affaire, c'est qu'il est utilisé en cette occasion par un juriste et non par un théologien. Voir Graz, UB, ms. 356, f. 11rv. Texte cité par M. J. CABLE, « Haec sancta and the continuity of judicial process... », p. 439.

64. M. J. CABLE, « Haec sancta and the Continuity of Judicial Process... », p. 440 ; ACC, t. IV, p. 309. Le texte anonyme qui présente très bien la position des Bourguignons est lui aussi intéressant : $A C C$, t. IV, p. 310.

65. Paris, BnF, ms. lat. 1485 I, f. 10 (voir supra, n. 20). C'est un texte bref, dont Martin John Cable a récemment analysé certains fragments : M. J. CABLE, «Cum essem in Constantie... » Raffaele Fulgosio..., p. 273-278.

66. Ibid., p. 272.

67. JeAn Gerson, Opera omnia, t. V, 653.

68. M. J. CABLE, «Cum essem in Constantie... » Raffaele Fulgosio..., p. 273.

69. J. Chiffoleau, " "Ecclesia de occultis non iudicat"... », p. 476. On analyse ici, tout particulièrement, les deux constitutions Ad reprimendum et Quoniam nuper est de l'empereur Henri VII, lors de sa descente en Italie. Sur les constitutions, voir Bartolo de Sassoferrato, Tractatus de constitutione Ad reprimendum, dans Opera, Venise, 1596, t. X, f. 95, et la glose 
Martin Porrée, si Sigismond condamnait la première assertion, il soutiendrait une idée contraire à Qui sint rebelles. Ce texte, amplement glosé qui abordait le thème du crimen laesae maiestatis, suggérait que les sujets pouvaient agir sans mandat pour punir ceux qui conspiraient contre la maiestas ${ }^{70}$. Bien qu'il existât en France une longue tradition d'application du droit romain, ramassée dans la formule rex in regno suo princeps est, dans le cas de Qui sint rebelles, la principale objection résidait dans le fait que Bartole et Balde bornaient l'usage de ce texte au domaine impérial ${ }^{71}$. Fulgosio soutenait, au contraire, que l'usage du droit romain par le roi de France suscitait son application au sein du royaume. Il ne pouvait y être appliqué pareillement que dans les affaires ecclésiastiques, sans pour autant que cela soit contraignant ni restrictif par rapport à l'autorité royale ou ecclésiastique ${ }^{72}$.

\section{Les Gersonistes battent en retraite}

Le 2 février 1416, Gerson prêchait Suscepimus Deus misericordiam tuam. Il s'y montrait abattu en voyant la vérité renversée et en ruines. Au sein du Concile, certains alléguaient aussi que les évêques ne devaient pas statuer sur des vérités de la foi et que les erreurs ne pouvaient être dénoncées

éditée dans le Corpus iuris civilis, Lyon, 1647, vol. Legum, col. 170-171. Sur ces textes, voir D. QuaGlioni, " "Fidelitas habet duas habenas". Il fondamento dell'obbligazione politica nelle glosse di Bartolo alle costituzioni pisane di Enrico VII », dans G. Chiтtolini, A. Molнo, P. SCHIERA éd., Origini dello Stato. Processi di formazione statale in Italia fra Medioevo ed età moderna, Bologne, 1994, p. 381-396 ; ID. «Rebellare idem est quam resistere. Obéissance et résistance dans les gloses de Bartole, à la constitution Quoniam nuper d'Henri VII (1355) », dans J. C. ZANCARINI et al. éd., Le Droit de résistance. XII ${ }^{e}$-XXe siècle, Paris, 1999, p. 35-46; K. Pennington, The Prince and the Law, 1200-1600. Sovereignty and Rights in the Western Legal Tradition, Los Angeles/Oxford/Berkeley, 1993, p. 165-202.

70. Pour le texte de Martin Porrée, voir Jean Gerson, Opera omnia, t. V, p. 486, et sur le consilium de Fulgosio voir M. J. CABLE, "Cum essem in Constantie... » Raffaele Fulgosio..., p. 275. L'auteur se montre ici particulièrement intéressé par les violations due process avalées par le texte.

71. M. J. Cable, "Cum essem in Constantie... » Raffaele Fulgosio..., p. 274-275. La maxime rex in regno suo princeps est avait une très longue tradition en France pour l'utilisation des concepts de plenitudo potestatis et superiorem non rescognoscens. À propos des origines de la maxime et sur ces concepts, voir F. CALASSO, "Origini italiane della formola "rex in regno suo imperator" », Rivista di storia del diritto italiano, 3 (1930), p. 213259 ; ID., I glossatori e la teoria della sovranità, Milan, 1957 ; S. Mochio ONoRy, Fonti canonistiche dell'idea moderna dello Stato, Milan, 1951 ; M. DAVID, La Souveraineté et les limites juridiques du pouvoir monarchique $d u X^{e}$ au Xv siècles, Paris, 1954, p. 57-58 ; B. BaSdeVAnt-GAudemet, «Plenitudo potestatis », Revue d'éthique et de morale, 227 (2003), p. 171-178 ; J. THÉRY, «Une hérésie d'État. Philippe le Bel, le procès des "perfides templiers" et la pontificalisation de la royauté française », Médiévales, 60 (2011), p. 157-186 ; J. KRYNEN, L'Empire du Roi...,p. 67-84. Sur l'application du crimen maiestatis sur le roi français par des juristes tels Blanot, Durand et Révigny, voir S. H. CutTLER, The Law of Treason..., p. 10-15.

72. M. J. CABLE, «Cum essem in Constantie... » Raffaele Fulgosio..., p. 276. 
que si ceux qui en étaient responsables étaient mentionnés ${ }^{73}$. Le jour même où Gerson prononça ce sermon, parurent ses Conclusiones de iure episcoporum. Il y abordait le sujet de l'autorité épiscopale dans la sanction et dans la détermination des questions de foi. Il prétendait que refuser aux prélats la faculté de déclarer hérétiques certaines propositions dérogeait à l'autorité épiscopale fondée sur le droit divin et légitimement prescrite dans la coutume. Cette manière de procéder, selon lui, irait à l'encontre des pratiques du Concile. Gerson se montre éloquent à propos de cette louable coutume, lorsqu'il affirme qu'à Paris, Oxford et Prague, le pouvoir épiscopal, de concert avec des studia generalia theologiae, a sanctionné des propositions hérétiques. Il doit pourtant reconnaître d'emblée qu'une décision prise de cette façon n'avait pas une portée universelle. En effet, le pape pouvait prendre une décision révoquant la sentence précédente, même si cela menaçait d'altérer le bon fonctionnement des instances hiérarchiques intermédiaires. Pour finir, Gerson revient sur les précédentes décisions du Concile qui avait entériné les condamnations prononcées conjointement par l'autorité épiscopale et les universités in Anglia et in Praga ${ }^{74}$.

Le 19 mars, il s'adresse au Concile, probablement en réponse à la lettre reçue du roi le 2 mars, dans laquelle il déclare nécessaire de s'opposer à une décision qui ferait fi de la justice ${ }^{75}$. En mai de la même année, il prononce un nouveau prêche Deus iudicium tuum regi da: il y fait référence au pouvoir royal français et place le roi au centre des débats ${ }^{76}$. Dans ce sermon, il se demande aussi de façon rhétorique comment il est possible que des experts en utrumque ius aient passé presque onze mois à discuter une doctrine hérétique et sacrilège comme s'il s'agissait d'une proposition potentiellement vraie ${ }^{77}$. Il se montre non disposé à accepter la décision de la commission conciliaire ni celle des trois cardinaux concernant l'appel.

C'est à cette époque qu'il écrit également les Octo regualae super stylo theologico, dans lesquels il revient sur le mode opératoire du Concile dans les questions de foi et où il propose de suivre un stylus theologicus au lieu d'un stylus iuridicus tel qu'il avait été développé dans les affaires de Wyclif et de Hus. Ce texte bref insiste sur la nécessité de privilégier les intérêts de Dieu, de la foi et de la reipublicae christianae, plutôt que ceux d'une singularis personae ${ }^{78}$. Sans le nommer expressément, Gerson faisait référence au duc de Bourgogne. Le conflit entre les deux camps au sein de

73. B. P. McGuIRE, Jean Gerson and the Last Medieval Reformation..., p. 255-256 ; JEAN Gerson, Euvres complètes, t. V, p. 538-546.

74. JeAn Gerson, Euvres complètes, t. VI, p. 174-176.

75. JeAn Gerson, Euvres complètes, t. X, p. 254-255, 540-542.

76. B. P. MCGUIRE, Jean Gerson and the Last Medieval Reformation..., p. 257-259 ; JEAN Gerson, Euvres complètes, t. V, p. 190-204.

77. JEAN GERSOn, ibid., p. 197.

78. ID., t. X, p. 260-262. 
la natio gallicana se poursuivit tout au long du mois de mai et c'est dans ce cadre que se répandit la rumeur selon laquelle l'affaire serait réglée après l'élection du nouveau pape. Face à cette possibilité, Gerson répondit qu'attendre jusqu'à l'élection ne ferait que remettre en question l'autorité du Concile lui-même ${ }^{79}$. Le 15 mai, dans une lettre dirigée à Sigismond, les trois cardinaux défendaient leur sentence et soumettaient au Concile leur décision au sujet des neuf assertions ${ }^{80}$. Le 7 juin, Gerson prêchait Spiritus Domini replevit orbem terrarum, et ne faisait nulle référence explicite au tyrannicide.

En avril 1416, après un séjour peu concluant à Paris, Sigismond rompait avec les Armagnacs et mettait le cap sur l'Angleterre pour y sceller une alliance ${ }^{81}$. Dans une dernière tentative, le contenu du texte - même s'il ne parlait pas du tyrannicide - recherchait peut-être la captatio benevolentiae de Sigismond afin d'obtenir son engagement dans la sanction des thèses de Jean Petit. Pourtant, depuis un certain temps, la politique impériale avait pris une direction opposée.

Le 4 juillet 1416, Gerson présenta un memorandum aux députés des quatre nations chargés de la causa, afin de relancer l'affaire. Devant l'impossibilité de parvenir à un accord, le 11 juillet, les membres de la Commission choisissaient de renvoyer l'affaire devant le Concile. Le 5 octobre, Gerson en appela une nouvelle fois au Concile afin de continuer les discussions, et quinze jours plus tard, les membres de la Commission de la foi rejetaient cet appel en raison de sa frivolité. Le 10 octobre, les lettres royales arrivées à Constance ne faisaient nullement référence à la question du tyrannicide et le 11 de ce même mois, Gerson recevait un sauf-conduit du roi dans le cas où il déciderait de quitter le Concile ${ }^{82}$.

Quoiqu'aucune solution définitive n'ait été trouvée, l'affaire Petit n'occupa plus une place prépondérante dans l'agenda du Concile. Dans une brève série de propositions datées du 28 février, Louis de Bavière expliquait pourtant pourquoi cette causa fidei ne devait pas être reportée après l'élection du nouveau pape. Selon lui, si l'affaire l'était, l'autorité du Concile pourrait être mise en question. En l'absence de solution définitive à propos des neuf assertions, le doute serait jeté sur la condamnation précédente de Quilibet tyrannus. Enfin, la sédition serait favorisée in omni policia ${ }^{83}$.

79. A. Coville, Jean Petit. La question du tyrannicide..., p. 544 ; JeAn Gerson, Opera omnia, t. V, p. 675.

80. JeAn Gerson, Opera omnia, t. V, p. 586, et p. 606-607 pour juin 1416.

81. S. ValleRY-RADOt, Les Français à Constance ..., p. 331. Le traité de Canterbury du 15 août 1419 scelle l'alliance entre Sigismond et Henri V. Sigismond se lasse de l'ambiguitté de la politique des Armagnacs et les accuse de ne pas avoir empêché l'abdication de Benoît XIII au cours de leur voyage à Narbonne. Le principal problème des Armagnacs consistait à rechercher leur autonomie face à Sigismond et à obtenir son appui contre les Anglais.

82. B. P. McGuire, Jean Gerson and the Last Medieval Reformation ..., p. 257-259; JeAN Gerson, CEuvres complètes, t. X, p. 546-547.

83. $A C C$, t. IV, p. 341. 
Le 8 janvier 1418, Pierre d'Ailly sollicita du pape récemment élu son intervention dans ce qu'il considérait comme une doctrine pestifère. Il est intéressant de noter qu' aux dires de Pierre d'Ailly, les Gersonistes gardaient toujours l'espoir que cette affaire serait traitée summarie et de plano ac sine strepitu et figura iudicii, comme ils le souhaitaient depuis le début ${ }^{84}$.

\section{Le tyrannicide : materia fidei et materia iuris}

Grâce à la thèse de Sophie Vallery-Radot, nous connaissons aujourd'hui beaucoup mieux la composition de la natio gallicana à Constance et les tensions provoquées en son sein par l'affaire Jean Petit ${ }^{85}$. Un des éléments à prendre en considération est le modus procedendi en cas d'hérésie savante. Pour Gerson et pour la majorité des Pères conciliaires, le fait d'en appeler fréquemment au Siège apostolique était associé au centralisme administratif curial qu'ils cherchaient à combattre et qu'ils accusaient d'être à l'origine du Schisme. L'appel omisso medio faisait du tort aux instances hiérarchiques intermédiaires. C'est pourquoi Gerson, comme pour l'affaire Jean Hus à Constance, s'efforçait de faire entériner par les Pères la décision prise conjointement par la corporation universitaire et le pouvoir épiscopal. Cette démarche visait assurément à faire également confirmer la sentence précédente de l'évêque de Paris. La façon de procéder dans les cas d'hérésie académique était cependant fortement enracinée dans une vision ecclésiologique de la structure constitutionnelle de l'Église, qu'il partageait avec plusieurs des Pères conciliaires ${ }^{86}$. Cependant, Gerson se gardait bien de rappeler que la condamnation des neuf assertions était loin d'avoir bénéficiée de l'unanimité de la corporation universitaire - et, de fait, la résistance de la Faculté de droit canonique, ainsi que celle de la natio picarda, sont bien connues ${ }^{87}$.

À ce stade, il est intéressant de signaler la réticence de la plupart des juristes à considérer les thèses de Jean Petit en tant que materia fidei. Bien que

84. ACC, t. IV, p. 351.

85. S. Vallery-Radot, Les Français à Constance..., p. 257-269. Cette étude prosopographique très intéressante sur la natio gallicana permet de constater que loin d'être unie, la natio était empreinte et marquée non seulement par les tensions entre les Armagnacs et les Bourguignons, mais aussi par celles entre universitaires et ambassadeurs du roi. Quoique 1'hypothèse centrale d'une consolidation d'une «identité française » au sein du Concile puisse sûrement être contestée historiographiquement, le propos est d'une grande utilité.

86. J. L. GAZZANIGA, «L'appel "omisso medio" au pape et l'autorité pontificale au Moyen Âge », Revue historique de droit français et étranger, 60/3 (1982), p. 395-414 ; S. PROVVIDENTE, «Clavis scientiae et clavis potestatis. La causa Hus entre le pouvoir épiscopal, universitaire et conciliaire », Studia Mediaevalia Bohemica, 3 (2011), p. 69-93.

87. Sur les purges qui ont affecté la natio picarde et la faculté de decretum à l'université de Paris, voir A. Coville, Jean Petit. La question du tyrannicide..., p. 551-552. 
le fait d'imposer un stylus iuridicus dans le traitement des assertions semble avoir été le fruit de la stratégie du camp bourguignon, ce fut également une conséquence de la façon dont Jean Petit avait, depuis le début, lui-même associé la tyrannie au crimen maiestatis, laissant ainsi la porte ouverte aux connotations juridiques revêtues par ce terme. Ceci peut peut-être s'expliquer par le caractère collectif de la rédaction de la Justification, dans laquelle le théologien Jean Petit semblait souvent s'exprimer plutôt comme juriste ${ }^{88}$. Au-delà des positions politiques conjoncturelles des participants aux débats sur le tyrannicide, il est clair qu'il existait une tension importante autour de ce problème, entre le discours théologique et le juridique. Corinne LeveleuxTeixeira a démontré l'existence d'un véritable drame herméneutique autour des neuf assertions de Jean Petit concernant le tyrannicide ${ }^{89}$. Nous croyons pourtant que les explications qui opposent expressément un discours moral d'ordre théologique sur la violation des principes de Non occides et Non perjurabis à un discours juridique, qui refuse de considérer le tyrannicide comme une question de foi, simplifient excessivement le problème ${ }^{90}$. Les discussions sur le tyrannicide à Constance semblent d'ailleurs être liées à la question juridique de l'exception. S'il est indéniable que les juristes avaient bien plus souvent affaire à ce type de difficulté que les théologiens, ces derniers abordaient également les conséquences de l'exception en se prévalant de concepts tels que celui de l'epikeia ${ }^{91}$. Ce qui différenciait alors les théologiens des juristes était sans doute l'évaluation des conséquences de l'exception. Dans le cas du tyrannicide, les premiers se montraient très réticents à accepter que des individus puissent prendre des décisions sur un cas exceptionnel, tel que statuer sur la conduite d'un tyran et procéder à son élimination. Du moins Gerson paraissait être particulièrement sensible à ces questions et s'y opposait sans une déclaration judiciaire, puisqu'il craignait les répercussions possibles tant en matière de politia séculière que de politia ecclésiastique ${ }^{92}$.

Au-delà des tensions entre discours théologique et juridique, il est important de souligner qu'il existait, au sein du discours théologique même, des points de vue distincts à propos des principes de l'exégèse

88. Sur le caractère collectif de la Justification, voir A. Coville, Jean Petit. La question du tyrannicide..., p. 123-126. Sur Guillaume Euvrie, voir É. Pellerin, « Un humaniste du temps de Charles VI : Guillaume Euvrie », dans EAD., Bibliothèques retrouvées. Recueil d'études publiées de 1938 à 1985, Paris, 1988, p. 437-456.

89. C. Leveleux-Teixeira, « Du crime atroce à la qualification impossible : les débats doctrinaux autour de l'assassinat du duc d'Orléans (1408-1418) », dans F. ForONDA, C. Barralis, B. SÈre éd., Violences souveraines au Moyen Âge. Travaux d'une école historique, Paris, 2010, p. 261-270 ; P. Von Moos, Öffentliches und Privates, Gemeinsames und Eigenes. Gesammelte Studien zum Mittelalter, Berlin, 2007, t. III, p. 165-194.

90. J.-P. Boudet et J. Chiffoleau, « Magie et construction de la souveraineté... », p. 44.

91. L. Buisson, Potestas und Karitas. Die päpstliche Gewalt im Spätmittelalter, Cologne/ Graz, 1958, p. 115.

92. Jean Gerson, CEuvres complètes, t. III, p. 304-305. 
biblique. D'un côté, les Pères conciliaires, et plus spécialement les Gersonistes, s'opposaient aux Bourguignons sur la manière d'interpréter le commandement Non occides. Selon ces derniers, le texte biblique ne pouvait être expliqué littéralement et c'est pourquoi ils en proposaient une lecture spirituelle. Les Gersonistes devaient par ailleurs affronter Jean Hus dont les thèses jugées par le Concile paraissaient trouver leur origine - malgré les démentis du maître tchèque - dans une lecture littérale de la Bible. Gerson devait catégoriquement affirmer que toute scientia theologica devait être fondée sur une lecture littérale des Saintes Écritures, sans pour autant tomber, ni dans l'erreur du littéralisme biblique de Hus, ni dans celle de l'herméneutique spirituelle des Bourguignons. Entre ces deux écueils, Gerson développa l'une des plus subtiles herméneutiques bibliques du bas Moyen Âge, échappant aux visions confessionnelles simplificatrices qui avaient cherché jusqu'alors des antécédents au principe de la sola scriptura ou de la tradition comme source d'autorité ${ }^{93}$.

Les contradictions dans la pensée de Gerson risquaient toutefois d'être dévoilées par son échec à faire condamner les neuf assertions. S'il n'était pas possible qu'un concile puisse se tromper en matière de foi, qu'était-il arrivé avec Jean Petit et sa condamnation à Constance ? Comment pouvait-il soutenir l'idée que le concile est la regula a spiritu sancto directa qui garantissait la vraie interprétation du texte biblique après ce qui s'était produit dans la causa Petit ? Qu'arrivait-il si on envisageait le fait qu'un concile puisse se méprendre en matière de foi ? Après l'expérience de Constance, Gerson revint sur certaines de ces questions dans son texte De examinatione doctrinarum, écrit en 1423, dans lequel il se vit obligé de remettre en cause un grand nombre des certitudes sur lesquelles reposait sa pensée ecclésiologique ${ }^{94}$.

Il existe différents points de vue expliquant l'obsession de Gerson pour la sanction des neuf assertions ${ }^{95}$. Comme nous l'avons suggéré, on peut soutenir que la réaction de Gerson s'explique, tout au moins partiellement,

93. Récemment exposé d'une façon très détaillé dans un article l'exégèse biblique de Gerson dans toute sa complexité par D. Z. Flanagin, « Making Sense of It All : Gersons's Biblical Theology », dans B. P. McGuire, A Companion to Jean Gerson, Leyde, 2006, p. 133178. Dans cet article, l'auteur effectue un retour historiographique sur cette question. Pour l'affaire de Jean Petit à Constance, voir l'indispensable K. Froelich, «Always to Keep the Literal Sense in Holy Scripture Means to Kill One's Soul : The State of Biblical Hermeneutics at the Beginning of the Fifteenth Century », dans E. R. MineR éd., Literary Uses of Typology from the Late Middle Ages to the Present, Princeton, 1977, p. 20-48; et S. PROVVIDENTE, «Clavis scientiae et clavis potestatis... », p. 69-93.

94. D. Z. FlanAGin, « Making Sense of It All... », p. 174.

95. D'une part, on a suggéré, d'une manière quelque peu naïve, que l'inimitié de Gerson envers le duc de Bourgogne serait due au fait que celui-ci lui aurait soustrait une prébende ecclésiastique ce qui l'aurait dès lors poussé à agir avec rancune à son encontre. D'autre part, on a également soutenu que les violences que Gerson avait personnellement endurées au cours de la révolte de Caboche l'auraient amené à identifier les thèses de Jean Petit comme la 
par le fait que sa vision des thèses de Petit secouait les fondements de sa pensée ecclésiologique et politique ${ }^{96}$. Nous croyons cependant qu'il serait non seulement nécessaire d'expliquer la préoccupation de Gerson pour la sanction du tyrannicide, mais aussi le silence des anti-Gersonistes, indépendamment de la conjoncture politique qu'ils défendaient. Pour une certaine historiographie, la défaite de Gerson et le fait que sa voix soit restée seule à dénoncer les thèses de Jean Petit semblent confirmer l'impression que, dans le contexte d'un pouvoir royal hautement sacralisé comme celui de la France, les débats sur le tyrannicide ne présentaient que peu d'intérêt, et c'est précisément cela qui aurait provoqué l'isolement de Gerson. Sans ignorer les immenses apports de cette historiographie sur les idées politiques du bas Moyen Âge français, nous pensons qu'elle ne s'est pas suffisamment arrêtée sur le crimen maiestatis et son rapport avec la construction du pouvoir royal ${ }^{97}$. La maiestas royale semblait paradoxalement exister par ce qui la blessait. En qualifiant juridiquement la tyrannie de crimen maiestatis, Jean Petit - ou le collectif qui avait écrit le texte de la Justification - ouvrait la porte à une qualification juridique qui, à la différence d'autres, était par nature dynamique et flexible, avec une tendance à toujours vouloir s'étendre à différents domaines. De la même manière que la majesté définie par gradus apparaît comme une grandeur impossible à mesurer et à circonscrire, les crimes perpétrés à son encontre sont eux aussi en perpétuelle expansion et impliquent autant la majesté divine que la majesté humaine à ses différents degrés ${ }^{98}$. Les crimes imputés au frère du roi, Louis d'Orléans, relevaient de cette logique expansive du crimen maiestatis et n'obéissaient pas seulement à une logique polémique bourguignonne à l'encontre du duc, ni ne reflétaient une mentalité laïque populaire et irrationnelle inscrite dans le discours théologique ${ }^{99}$. En raison de ces caractéristiques, le crimen maiestatis ouvrait

source de la discorde civile de cette époque : A. FrenKEn, « Die Erforschung des Konstanzer Konzils...»,p. 201.

96. Ibid.

97. J. KRYNEN, Idéal du prince et pouvoir royal en France à la fin du Moyen Âge, Paris, 1981, p. 1985. Sur l'importance du crimen maiestatis dans la construction du pouvoir souverain, voir J.-P. Boudet et J. ChiFfoleAu, « Magie et construction de la souveraineté... », p. 46 : «Le recours aux pratiques magiques et les dénonciations auxquelles elles donnent lieu ont des rapports étroits avec la construction de la souveraineté. Pas seulement, encore une fois, parce qu'elles peuvent être instrumentalisées par tel ou tel puissant dans telle ou telle circonstance, mais parce qu'elles mettent presque toujours en cause, et contribuent à faire vivre la majesté divine et humaine. »

98. J. Chiffoleau, « Amédée VIII ou la majesté impossible », dans B. Andenmatten, A. Paravicini Bagliani éd., Amédée VIII-Felix V, premier duc de Savoie et pape (1383-1451), Lausanne, 1992, p. 19-49 ; ID., « Note sur la bulle Vergentis in senium. La lutte contre les hérétiques du Midi et la construction des majestés temporelles », Innocent III et le Midi, Cahiers de Fanjeaux, 50 (2015), p. 89-144.

99. J.-P. Boudet et J. Chiffoleau, « Magie et construction de la souveraineté... », p. 4546 ; B. Guenée, La Folie de Charles VI..., p. 88-98. 
la porte à l'exception en matière procédurale. Entre autres choses, c'est justement autour du problème de l'exceptionnalité que les juristes et les théologiens se sont affrontés à Constance.

En se refusant à discuter du tyrannicide sur un plan juridique, Gerson, ainsi que tous ceux qui cherchaient à faire condamner les thèses de Jean Petit, avaient perdu la possibilité de se prévaloir de ce qui aurait pu être un solide argument contre le duc de Bourgogne. Ne pouvait-on pas considérer que l'assassinat du duc d'Orléans était également un crimen maiestatis, puisqu'en passant à l'acte sine declaratione iudiciaria, on s'en était pris à une pars corporis regis, dans la mesure où, non seulement il s'agissait de son frère, mais aussi d'un de ses principaux conseillers ? Le texte Quisquis se montrait plus qu'éloquent envers ceux qui s'en prenaient aux magistrats et aurait également pu être utilisé pour qualifier l'assassinat du duc de crime contre la majesté royale ${ }^{100}$. Récemment, Jean-Patrice Boudet et Jacques Chiffoleau se demandaient comment il était possible que Jean sans Peur passe outre la justice du roi lui-même en commettant le meurtre du duc d'Orléans. Qui avait attenté contre la maiestas ? Jean sans Peur ou le duc d'Orléans ${ }^{101}$ ? Dans la détermination et dans la décision de l'exceptionnalité du crime contre la majesté, se jouait un rapport de forces entre les ducs de Bourgogne et d'Orléans. Les incessantes disparitions du roi du devant de la scène laissaient justement le champ libre pour que d'autres - en l'occurrence le duc de Bourgogne - définissent de quelle façon et par quels crimes cette même maiestas royale était blessée. Il est intéressant de constater que la construction de la majesté royale ne s'effectue pas totalement en dehors du droit. Au-delà de l'affirmation de violence déclenchée par Jean sans Peur avec l'assassinat du duc d'Orléans, la qualification juridique de ce meurtre en tant que crimen maiestatis était essentielle. La Justification n'était pas une simple justification du crime, mais plutôt un enjeu pour déterminer l'instance souveraine qui décidait de l'exceptionnalité dans le crimen maiestatis. Le duc de Bourgogne avait non seulement triomphé dans la via facti, mais également de iure. Aussi la détermination du crimen maiestatis, tout en continuant d'être considérée comme un acte juridique, était-elle nécessairement associée à un acte fondateur enraciné dans la violence. On a souvent voulu voir dans la Justification de Jean Petit un simple texte de propagande, écrit dans le but de légitimer le meurtre de Louis d'Orléans, sans approfondir la logique juridique du texte. Ce n'est pas un hasard si les auteurs élaborèrent leurs argumentations autour de la catégorie juridique du crimen maiestatis. Le fait que les Gersonistes refusent de débattre à Constance dans les termes

100. Cette loi, initialement promulguée par les empereurs Arcadius et Honorius, en 397 , fut ensuite incorporée au Codex Theodosianus et finalement au Codex Justinianus. Il y était établi que celui qui attaquerait les conseillers de l'empereur commettait un crimen maiestatis, puisque ipsi pars corporis nostri sunt (Cod.9.8.5).

101. J.-P. Boudet et J. Chiffoleau, « Magie et construction de la souveraineté... », p. 47. 
avancés par les juristes semble être en lien avec les dangers que représentait la décision individuelle sur le cas exceptionnel. En excluant de discuter en ces termes, les Gersonistes avaient perdu la possibilité de faire valoir un argument qui aurait pu sérieusement remettre en question la construction juridique de Jean sans Peur après l'assassinat.

Pour finir, dans le contexte politique du Concile, tout paraissait conspirer contre une sanction des neuf assertions en tant que materia fidei. L'affaire Petit menaçait de diviser la natio gallicana et, dans un même temps, la condamnation du tyrannicide n'était pas clairement une priorité pour la natio anglicana, étant donné qu'Henri V était monté sur le trône en éliminant Richard II. Au sein de la natio germanique, le conflit entre la couronne polonaise et l'ordre Teutonique n'offrait pas non plus un environnement propice pour discuter ces questions. Dans sa Satira, Johannes Falkenberg défendait d'ailleurs ouvertement le tyrannicide du roi de Pologne, puisqu'il ne reconnaissait pas sa conversion au christianisme ${ }^{102}$.

L'histoire de la causa Petit à Constance doit être réévaluée à la lumière des récentes recherches sur la natio gallicana. Au moment d'expliquer le rôle joué par Gerson dans cette affaire, il importe aussi de l'analyser dans le contexte de l'hérésie savante de son époque, dans celui de ses formes procédurales particulières. D'une part, Jean Gerson, dans la causa Petit, cherchait à faire confirmer par les Pères conciliaires - à l'instar d'autres cas de la causa fidei - la sentence déjà prononcée conjointement par la corporation académique et par le pouvoir épiscopal. Face à ces requêtes, les Bourguignons s'obstinèrent à soutenir que cette coutume dans le traitement de l'hérésie académique n'avait aucun fondement juridique solide et que, de fait, elle menaçait de toucher au privilège du Siège apostolique dans la détermination des questions de foi et d'altérer la hiérarchie ecclésiastique. Une étude comparative avec d'autres cas de causa fidei (Wyclif, Hus, Jérôme de Prague, Falkenberg) promet d'être un terrain fertile pour de nouvelles investigations. D'autre part, les débats au sein du discours théologique autour de l'herméneutique biblique semblent eux aussi avoir joué un rôle important dans le déroulement des discussions sur les neuf assertions.

Pour finir, nous pouvons constater, à partir de l'analyse des débats à Constance, l'existence d'une tension dans la question de la défense du tyrannicide entre le discours juridique et le discours théologique de Gerson. Les Gersonistes n'ont pas réussi à utiliser un argument qui aurait pu sérieusement remettre en question la construction juridique de Jean sans Peur après l'assassinat dans la mesure où l'assassinat du duc d'Orléans aurait également pu être présenté comme un crimen maiestatis parce qu'il

102. A. FrenKEN, « Die Erforschung des Konstanzer Konzils... », p. 207-238. Les rapports entre l'affaire Petit et l'affaire Falkenberg mériteraient une étude détaillée. 
constituait une attaque à une pars corporis regis. C'est dans la décision de l'exceptionnalité du crime contre la majesté que se jouait un rapport de force entre le duc de Bourgogne et le duc d'Orléans. La via facti et la via iuris semblent avoir été beaucoup plus intimement liées que les historiens n'ont bien voulu l'accepter ${ }^{103}$.

\section{Sebastián Provvidente - UBA-CONICET}

\section{Stylus theologicus et iuridicus : la causa Jean Petit à Constance (1414- 1418) et les débats sur le tyrannicide}

Dans ce texte on analyse les discussions entre théologiens et juristes à propos de la Justification du tyrannicide de Jean Petit dans le cadre du Concile de Constance (1414-1418). Au-delà des positions politiques conjoncturelles des participants aux débats sur le tyrannicide, il est clair qu'il existait une tension importante autour de ce problème, entre le discours théologique et le juridique. L'exclusion des Gersonistes des arguments des juristes était en lien avec les dangers que représentait la décision individuelle sur le cas exceptionnel. En excluant de discuter en ces termes, les Gersonistes avaient perdu la possibilité de faire valoir un argument qui aurait pu sérieusement remettre en question la construction juridique de Jean sans Peur après l'assassinat de Louis d'Orléans.

concile de Constance, procédure, stylus theologicus, stylus iuridicus, tyrannicide

\section{Stylus theologicus et iuridicus: Jean Petit in Constance (1414-1418) and the debates on tyrannicide}

In this text, we examine the debate between theologians and jurists on Jean Petit's Justification of tyrannicide, in the context of the Council of Constance (1414-1418). Beyond the political and circumstantial positions of the participants in the debates on tyrannicide, there was a very clear, important tension between theological and juridical discourse on this matter. The exclusion of the Gersonists from juridical arguments was related to the risks associated with individual decisions in this exceptional case. Through their exclusion from discussion, the Gersonists had lost the opportunity to use an argument that might have seriously questioned John the Fearless' juridical construction, after the assassination of Louis of Orléans.

council of Constance, procedure, stylus theologicus, stylus iuridicus, tyrannicide

103. Une version préliminaire de ce texte a été présentée dans le cadre du séminaire de Jacques Chiffoleau à l'EHESS à Paris en mai 2016. Un grand merci d'avoir été invité à y participer et merci également pour les commentaires, toujours intéressants, des participants, notamment ceux de Jean-Patrice Boudet. 
